\title{
VARIA
}

\section{NUEVAS OBRAS DE CARLOS DE HAES Y DE AURELIANO DE BERUETE}

El objeto de estas líneas es el de dar a conocer y catalogar cuatro nuevas obras de Carlos de Haes (Bruselas, 1826-Madrid, 1898) y otras dos de Aureliano de Beruete (Madrid, 1845Madrid, 1912). Tres de las de Haes pertenecen a colecciones particulares madrileñas y la otra ha aparecido en el comercio alicantino; mientras que las dos de Aureliano de Beruete son de colección particular madrileña. Comenzaremos este análisis por las de Haes, tanto por razones cronológicas como por haber sido este pintor maestro de Beruete.

La primera de ellas pertenece a la colección Rudolf Gerstenmaier (Madrid), y es un óleo sobre lienzo, de $0,35 \times 0,65 \mathrm{~m}$., firmado en rojo en el ángulo inferior izquierdo "C. de Haes", y que por representar un bello paisaje de los llamados de alta montaña, que no podemos identificar, lo titularíamos Vista de cordillera (Fig. 1). Casualmente, ya nos hemos ocupado en otras ocasiones recientes de este tipo de paisajes de Haes ${ }^{1}$, por ser cuadros de esa temática la mayoría de los que han llegado a mis manos últimamente, lo que, sin duda, nos permite establecer mejor comparaciones y diferencias. De ellos, especialmente dos, con asuntos de paisajes de alta montaña, poseen una estructura compositiva muy similar a la de este que analizamos, como veremos, y de los cuales uno, que ya estudiamos en su día ${ }^{2}$ y que titulamos entonces Los Picos de Europa (?) (Fig. 2), pertenece también a la colección Rudolf Gerstenmaier, mientras que el otro, que titularemos Paisaje de alta montaña con río y pastora (Fig. 3), está en una colección particular madrileña, y será objeto también de nuestro estudio a continuación de este primero.

En efecto, la composición del cuadro que traemos (Fig. 1) reserva casi un tercio de ella a los celajes y, prácticamente, los otros dos tercios restantes al paisaje en sí, al igual que ocurre en los otros dos cuadros (Figs. 2 y 3). Y, por lo que se refiere a la organización del paisaje, también hay una gran similitud estructural entre los tres. En el paisaje que estudiamos (Fig. 1), un escarpado valle discurre entre dos promontorios rocosos (que actúan como pantallas de referencia espacial, junto con los árboles, entre los primeros y últimos términos del cuadro), pertenecientes a las estribaciones de las imponentes montañas, cargadas de nieve, que se destacan, entre la brillantez calinosa de la atmósfera, al fondo central de la composición. Muy si-

\footnotetext{
${ }^{1}$ Arias Anglés, E. «Sobre tres paisajes de alta montaña de Haes», Archivo Español de Arte, t. LXXV, núm. 297, 2002, p. 57; Arias Anglés, E. «Miscelánea de pintura decimonónica», Archivo Español de Arte, t. LXXVI, núm. 304, 2003, pp. 407, y 413 a 415; Arias Anglés, E. «Nuevas obras de Jenaro Pérez Villaamil y Carlos de Haes», Archivo Español de Arte, t. LXXVII, núm. 306, 2004, pp. 177 y187 a 190.

${ }^{2}$ Arias Anglés, E. «Sobre tres paisajes de alta montaña de Haes», Archivo Español de Arte, t. LXXV, núm. 297, 2002, pp. 60-61.
} 


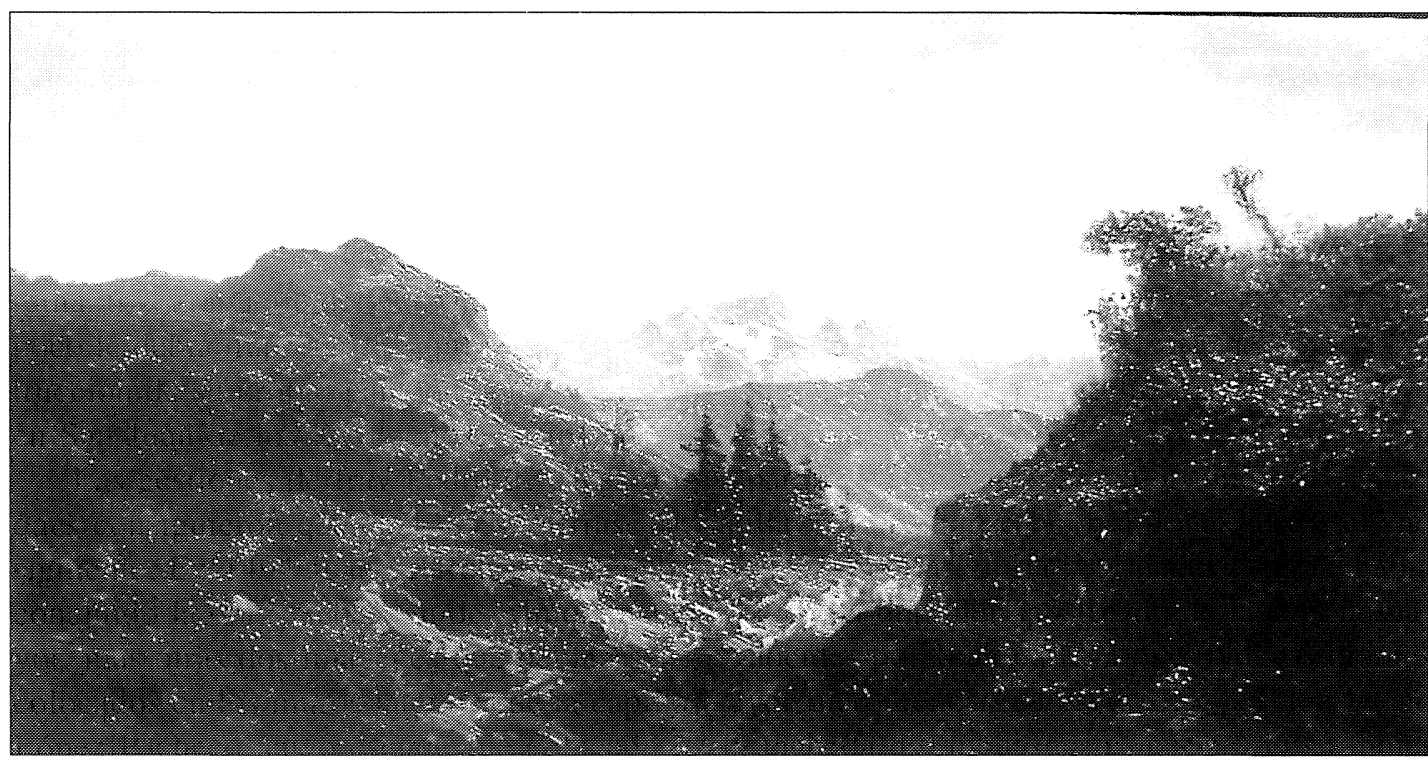

Fig. 1. Carlos de Haes: Vista de cordillera. Óleo/lienzo. Madrid. Colección Rudolf Gerstenmaier.

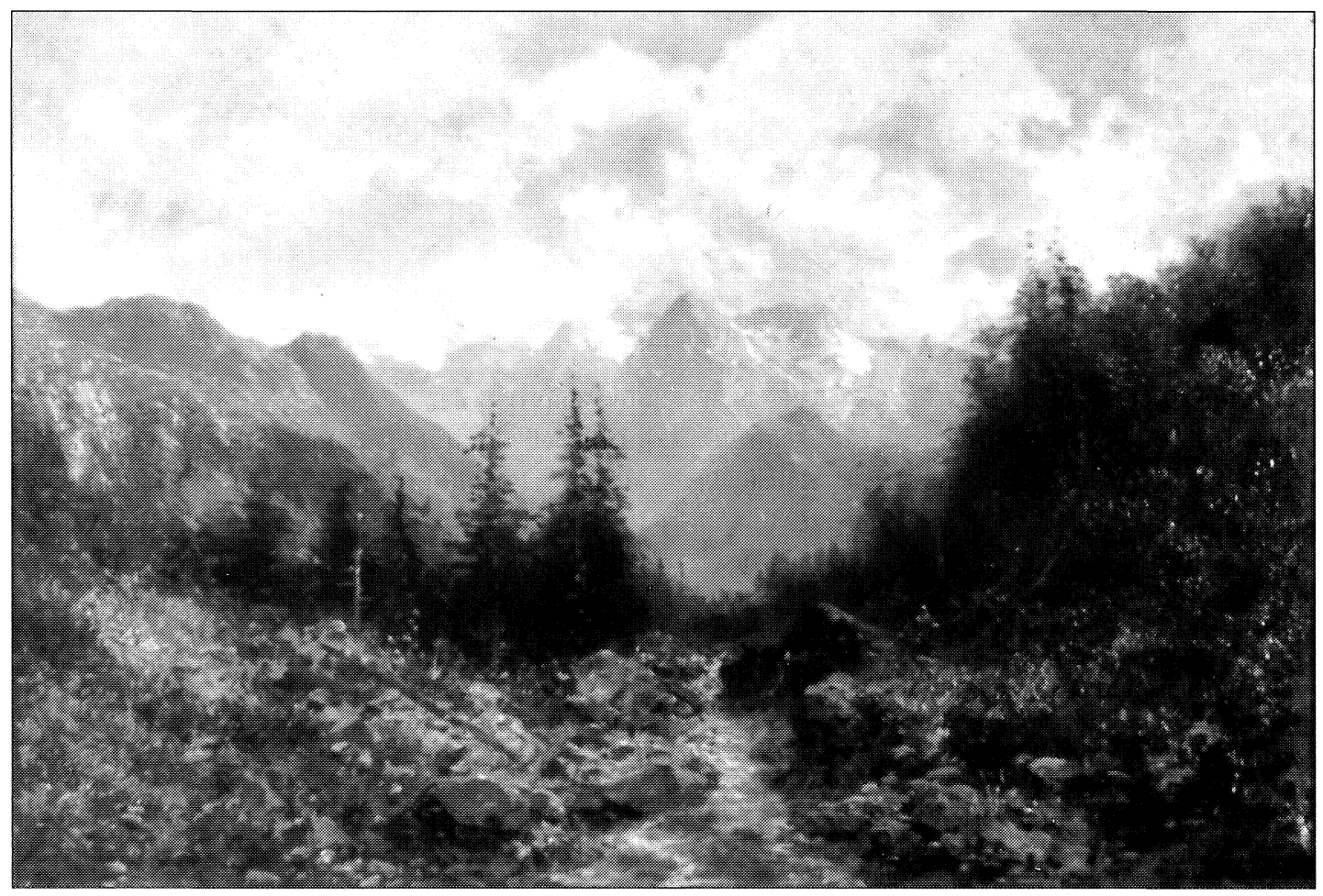

Fig. 2. Carlos de Haes: Los Picos de Europa (?). Óleo/lienzo. Madrid. Colección Rudolf Gerstenmaier.

AEA, LXXVIII, 2005, 311, pp. 297 a 331 
milar disposición tienen también los elementos geológicos y vegetales que conforman los otros dos cuadros (Figs. 2 y 3), con ligeras variantes respecto a la estructura del que estudiamos.

Pero el que guarda mayor similitud compositiva y estilística de esos dos con el cuadro que analizamos es, sin duda, el que titulamos Los Picos de Europa (?) (Fig. 2). En efecto, una simple ojeada comparativa basta para corroborarlo: casi idéntica estructura compositiva en ambos; las únicas diferencias estriban en que en el que estudiamos (Fig. 1) se acusan más, por su volumen y fuerte sombreado, los promontorios que actúan a modo de pantallas de referencia espacial en los términos medios de la composición, mientras que en el otro cuadro (Fig. 2) el abrupto valle central, presente en ambos cuadros, subraya más aquí su carácter de eje de la composición mediante la adición de un riachuelo que discurre desde el fondo al primer plano. Por lo demás, pocas diferencias esenciales; incluso podríamos decir que son casi idénticos los dos grupos de abetos o pinos de alta montaña que se sitúan hacia el centro de ambas composiciones, teniendo no sólo iguales formas, sino estando también realizados con la misma técnica.

La factura detallada y minuciosa del cuadro (Fig. 1), su perfecto acabado, a pesar de la soltura de pincelada, nos indican que se trata, sin duda, de una obra realizada en el taller del artista, sobre algún boceto tomado del natural, forma esta de trabajar habitual no sólo en Carlos de Haes, sino prácticamente en casi todos los pintores del realismo europeo del siglo XIX. Este hecho es importante a la hora de intentar dar una datación al cuadro, pues de haberse tratado de una obra abocetada, esto nos permitiría pensar que, al ser tomada directamente del natural, y ser un asunto de alta montaña, pudiera fecharse, sin duda, entre 1872 y 1875 , período en el que se produce, básicamente, el descubrimiento de los asuntos de alta montaña por parte de Haes, probablemente por sugerencia de su discípulo Aureliano de Beruete (influido ya entonces por el ideario del Colegio Internacional y de Giner de los Ríos), cuando los dos fueron a pintar a Asturias, Santander, Vasconia y Navarra, descubriendo entonces para su pintura los Picos de Europa y los Pirineos ${ }^{3}$.

Pero, a pesar de ser una obra de estudio y no un boceto tomado directamente del natural, como muy probablemente se trate de un paisaje de alguna de esas cordilleras, que nos es imposible de precisar, y como el interés de Haes por los paisajes de alta montaña se centra precisamente en torno a esos años de entre 1872 y 1875, unido todo ello a que el estilo pictórico del cuadro coincide con el que Cid Priego estableció para la que denominó su segunda etapa pictórica, fechable entre 1870 y 1880 , en que utiliza un colorido más jugoso y una factura más suelta que en su primera etapa ${ }^{4}$, podríamos resolver de esta concatenación de hechos que el cuadro fue pintado, muy probablemente por las fechas de entre 1872 y 1880 .

El segundo cuadro de Haes que traemos, y que ya nos ha servido de referencia comparativa con este primero, es el que hemos titulado Paisaje de alta montaña con río y pastora (Fig. 3 ), que es un óleo sobre lienzo de 0,89 × 1,465 m., firmado en el ángulo inferior derecho «C. de Haes», perteneciente, como ya hemos dicho, a una colección particular madrileña. La técnica de este cuadro manifiesta, igualmente, todas las características estilísticas y técnicas representativas de la producción pictórica de Haes; como el sabio juego de colores complementarios que utiliza, sin abandonar los grises y los negros, dividiendo mucho la pincelada, conformando así una tonalidad aparentemente uniforme a base de diverso colorido, aunque sin dar el paso definitivo hacia el impresionismo, consistente en aplicar toques yuxtapuestos de color puro. Y, al igual que en el cuadro anterior, su factura detallada y minuciosa, su perfecto acabado, nos indican también claramente que se trata de una obra realizada por el pintor en su

\footnotetext{
${ }^{3}$ Pena, M.C. El paisaje español del siglo xIx: del naturalismo al impresionismo, Universidad Complutense, Madrid, 1982, pp. 195, 198 y 199.

${ }^{4}$ Cid Priego, C. Aportaciones para una monografía del pintor Carlos de Haes, Instituto de Estudios Ilerdenses (CSIC), Lérida, 1956, pp. 18, 21 y 22.
} 
taller, sobre algún esbozo o apunte tomado en alguna sesión directa ante la naturaleza. Este hecho es lo que la diferencia bastante, estilística y técnicamente, de los numerosos apuntes abocetados que realizaba Haes del natural y que le servían luego para componer cuadros perfectamente acabados en su estudio; bocetos estos que se caracterizan por una técnica más suelta, de gran frescura y espontaneidad, pero no concebidos por el artista como tales cuadros, y de los que se conservan muchos en diversos museos y también en colecciones privadas, y a los que hoy día, por su perfección y aspecto casi preimpresionista, les damos el valor de cuadros en sí. Como ya hemos dicho, esta manera de proceder era común a todos los pintores del naturalismo europeo, para quienes la naturaleza era fundamental, pero no pintaban ante ella los cuadros definitivos, sino que eran reelaborados en el taller por medio de apuntes tomados al aire libre. De aquí que los cuadros de Haes realizados en su taller, posean una composición y un acabado, generalmente, de un bello academicismo, como es el caso de este hermoso cuadro que presentamos.

Por lo que respecta al tema de la obra, éste nos indica claramente que se trata también de un asunto de paisaje de alta montaña, temática con la que, como hemos dicho, entró en contacto Haes a partir de 1872, cuando fue a pintar en verano a Santander y Vizcaya, descubriendo así los paisajes del norte de España, tan semejantes en climatología y atmósfera a los de su tierra natal a que estaba tan acostumbrado ${ }^{5}$. Es pues en el período que discurre entre 1872 y 1875, según hemos visto, en el que se inscribe básicamente el interés de Haes por los paisajes de alta montaña. Y es, por tanto, dentro de ese período en el que podemos situar el origen de este cuadro. Pero, al igual que sucede con el cuadro anterior, al tratarse de una obra realizada en su estudio y perfectamente acabada, pudiera muy bien haber sido pintada por el artista en fechas muy posteriores a esas que indicamos, utilizando para su composición bocetos tomados entonces «in situ», y que él conservaría en su estudio.

Así pues, al tratar de fechar el cuadro, nada se opone a que procedamos siguiendo el mismo razonamiento que aplicamos al cuadro anterior, por presentar la misma problemática; es decir, que con la precaución que corresponde tomar - por tratarse de un cuadro compuesto en el taller y no directamente del natural-, como no es descabellado pensar que se trate de algún paisaje de la cordillera Cantábrica o de los Pirineos, que Haes anduvo pintando del natural entre esos años de 1872 y 1875 , y como el estilo pictórico del cuadro responde a las características estilísticas y técnicas que Cid Priego señaló para la segunda etapa pictórica del artista, que fechó, como decimos, entre 1870 y 1880 , podríamos deducir de todo ello que el cuadro fuese pintado, con cierta posibilidad, entre 1872 y 1880 , al igual que hemos establecido para el cuadro anterior de la colección Gerstenmaier.

La tercera de las obras de Haes que presentamos, perteneciente también a una colección particular madrileña, la hemos titulado Paisaje montañoso con río y cabaña (Fig. 4), y es un óleo sobre lienzo de $0,89 \times 1,31 \mathrm{~m}$., firmado y fechado en el ángulo inferior izquierdo: «C. de Haes / 1887».

Se trata, al igual que en los cuadros anteriores, de un paisaje con asunto de alta montaña, poseyendo también una estructura compositiva muy semejante a la de aquellos dos; un estrecho y escarpado valle de alta montaña, por el que discurre un riachuelo que viene del fondo de altas cumbres nevadas hacia el primer término, entre agrestes orillas con pinos y vegetación de altura. E igualmente es válido también en este caso todo lo que, al respecto, dijimos antes para aquellos dos. O sea, que también se trata de un cuadro realizado por el pintor en su estudio, sobre apuntes tomados del natural entre 1872 y 1875 muy probablemente, cuando entonces se despertó su interés por este tipo de paisajes en su visita a la cordillera Cantábrica y los

${ }^{5}$ Pena, M.C. Op. cit. nota 3, p. 195. 


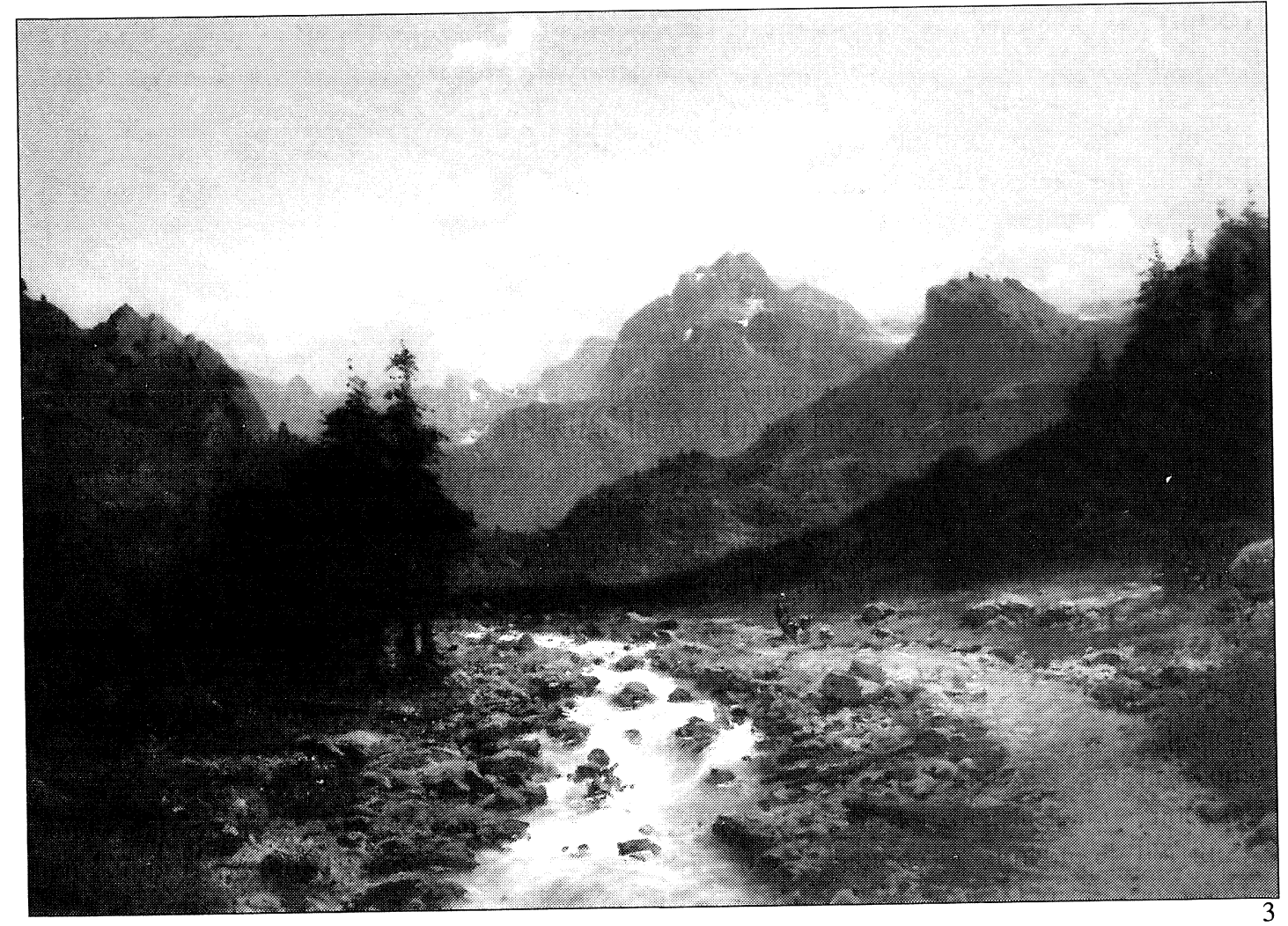

4

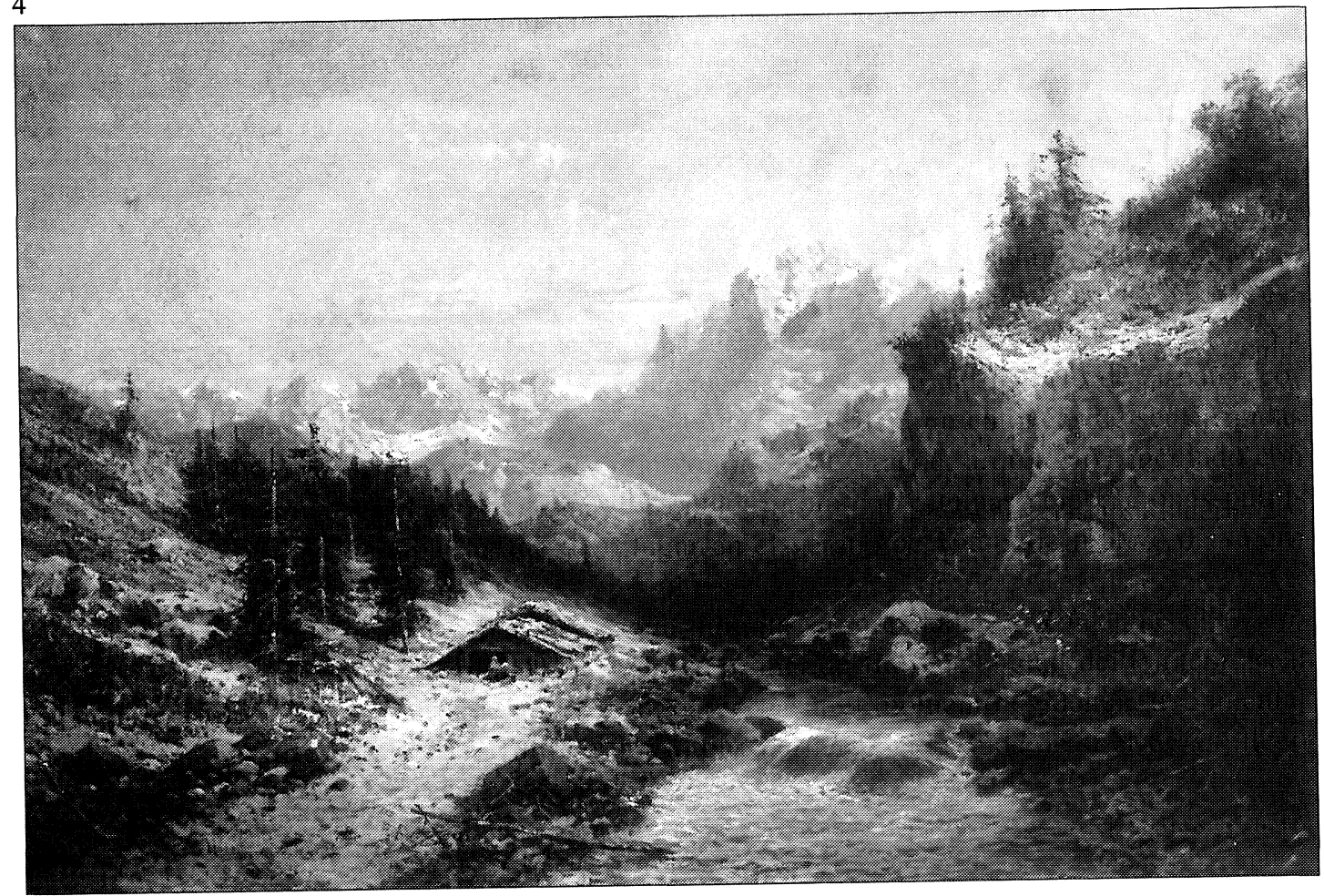

Fig. 3. Carlos de Haes: Paisaje de alta montaña con río y pastora. Óleo/lienzo. Madrid. Colección particular. Fig. 4. Carlos de Haes: Paisaje montañoso con río y cabaña. Óleo/lienzo. Madrid. Colección particular.

AEA, LXXVIII, 2005, 311, pp. 297 a 331 
Pirineos, pues estamos ante una obra meditadamente compuesta y perfectamente acabada. Es decir, que este cuadro, aunque realizado en el taller del pintor en 1887, como figura en el lienzo, sin embargo está compuesto, muy probablemente, sobre apuntes del artista tomados del natural en los Picos de Europa o en los Pirineos por esas fechas indicadas. Y más si tenemos en cuenta que la carrera artística de Haes quedó prácticamente paralizada poco tiempo después de pintarse este óleo, en 1890, debido a la enfermedad que ocho años más tarde acabaría con su vida ${ }^{6}$.

La fecha y el estilo del cuadro coinciden en indicarnos que esta obra pertenece a la que se ha denominado su tercera, y última, etapa ${ }^{7}$, en la que, debido, posiblemente, a las desgracias familiares, Haes abandona la alegría y brillantez de colorido que caracterizó a su segunda etapa, para adoptar un color menos brillante, pero de mayor transparencia, mostrando más afición por las nieblas y los tintes apagados del norte. Renuncia al gusto por la luz meridional, pero adoptando, en cambio, un vigoroso efecto, un colorido jugoso y una factura excelente y suelta.

La cuarta, y última, de las obras de Haes que traemos, aparecida recientemente en el comercio de Alicante, es un espléndido dibujo al carboncillo sobre papel pegado a soporte de madera, de buenas dimensiones, $0,72 \times 1,05 \mathrm{~m}$., y que se halla firmado en el ángulo inferior derecho, en clarión, «C. de Haes». Le damos el título de Paisaje lacustre de Vriesland (Holanda) (Fig. 5), según la identificación que del mismo hacemos más adelante en este estudio. Representa un paisaje lacustre en un día de borrasca: al primer término tenemos la vegetación de juncos y otros matorrales y hierbas que pueblan la orilla de la laguna, movidos violentamente por un fuerte ventarrón de tormenta; detrás de ella vemos la superficie acerada de las aguas, debido al reflejo de la apagada luz que se trasluce entre las nubes menos densas de un obscuro celaje tormentoso, que son violentamente arrastradas por el fuerte vendaval en un cielo cargado de presagios de lluvia; al fondo derecha, cerca de la orilla, se vislumbra una densa y sombría masa boscosa, que acentúa el aspecto tormentoso y de inminente chaparrón que domina en la composición.

Es de esas pocas obras de Haes en que podríamos decir que el protagonista de la misma no es el paisaje en sí, sino la sensación de tempestad que, magistralmente captada por el pintor, nos ha sabido representar, con gran realismo, en la composición. Es más difícil transmitir una sensación atmosférica por medio del dibujo que por el óleo o la acuarela, pero, a pesar de ello, aquí el protagonista es el fenómeno meteorológico en sí; la sensación atmosférica de un día de tempestad. Y todo ello, sin otro tipo de colorido que la monocromía que proporciona un lápiz de carboncillo y unos toques de gouache blanco al centro para avivar el reflejo de la luz en las aguas.

Realmente el dibujo es hermoso y ejemplifica la gran calidad que como dibujante poseía Haes (aprendida de su maestro el paisajista belga J. Quinaux ${ }^{8}$ ), detallando al primer término los diferentes tipos de matorrales de las orillas con unos trazos precisos, perfectos, así como magistralmente también difumina las formas a medida que se pierden en la cargada atmósfera de la distancia. Pero estos detalles técnicos no pasarían de ser más que bellos elementos estáticos, mudos, si no fuese por la enorme vida que adquieren bajo la densa atmósfera que preludia la tempestad, admirablemente captada por el lápiz del pintor. La atmósfera da vida al cuadro, el viento mueve vegetación y nubes, dando gran dinamismo a la composición.

Es difícil precisar con exactitud, por tratarse precisamente de un dibujo, a qué época o período de la obra de Haes pertenece. Cid Priego que, como decimos, dividió en tres períodos la

\footnotetext{
${ }^{6}$ Arias Anglés, E. Tres grandes maestros del paisaje decimonónico español: Jenaro Pérez Villaamil, Carlos de Haes y Aureliano de Beruete, catálogo exposición, Centro Cultural del Conde Duque, Ayuntamiento de Madrid, 1990, p. 255.

${ }^{7}$ Cid Priego, C. Op. cit. nota 4, p. 22.

${ }^{8}$ Ibidem, p. 17.
}

AEA, LXXVIII, 2005, 311, pp. 297 a 331 


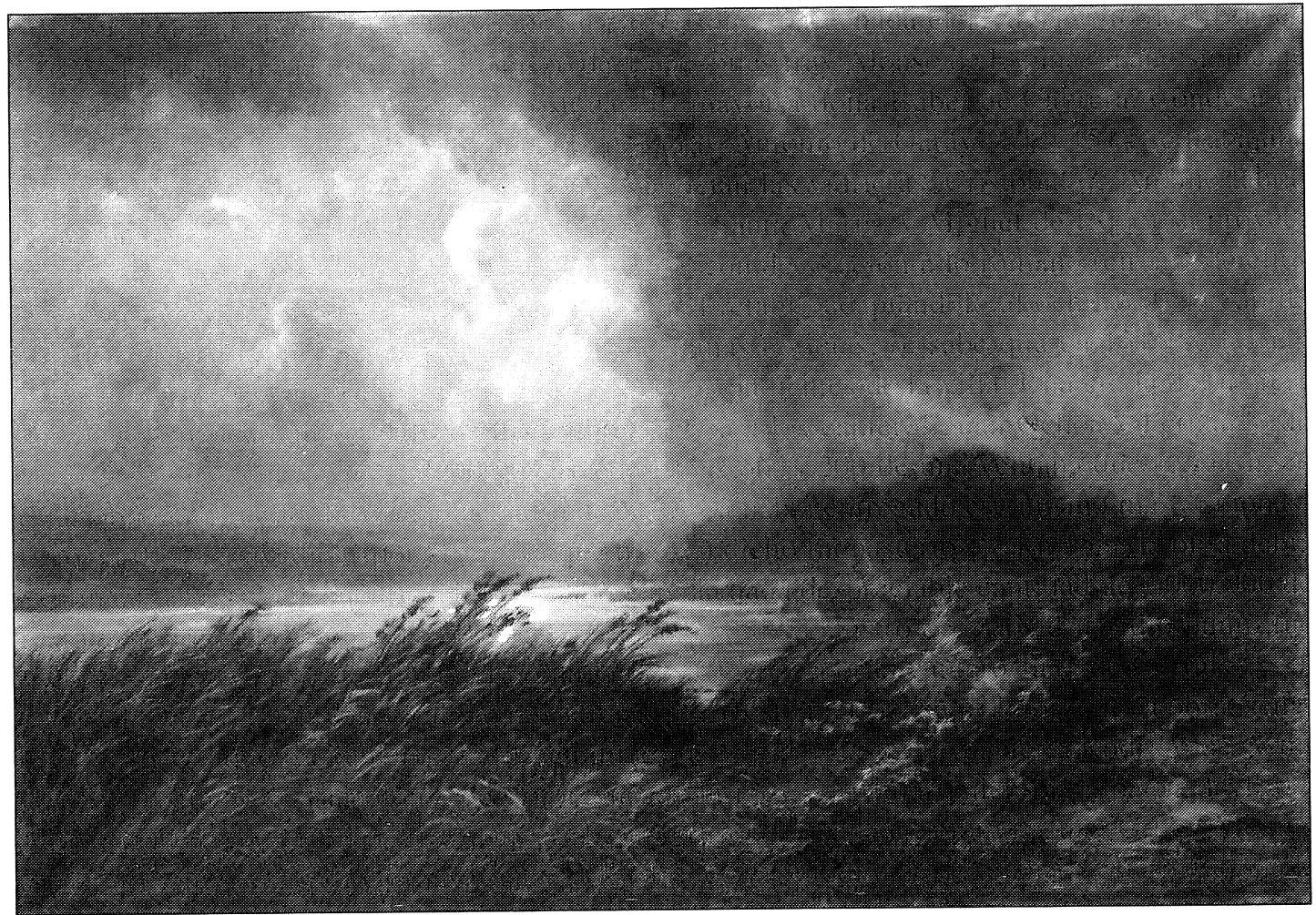

Fig. 5. Carlos de Haes: Paisaje lacustre de Vriesland (Holanda). Dibujo al carboncillo, con toques de clarión, sobre papel pegado a soporte de madera. Alicante. En el comercio.

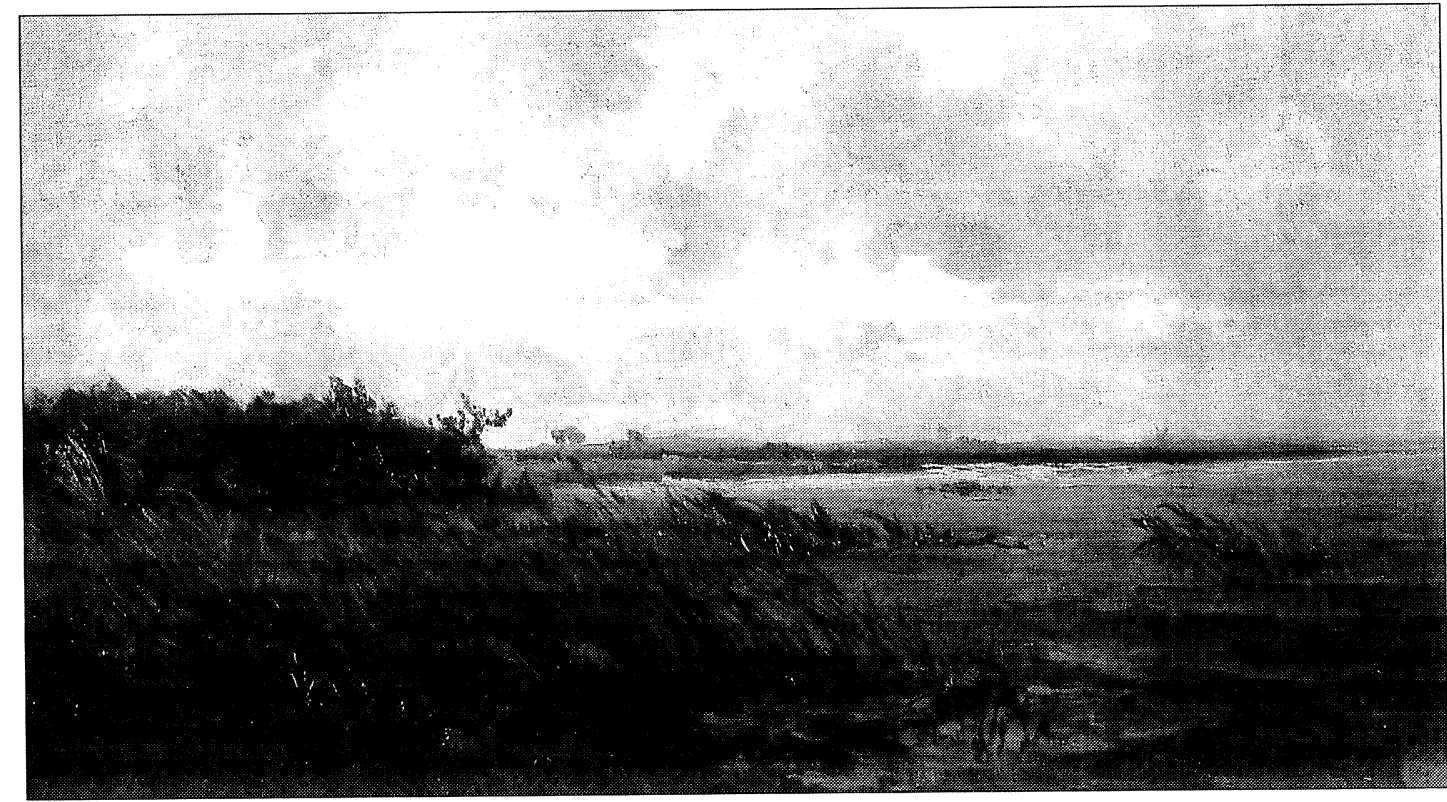

Fig. 6. Carlos de Haes: Un canal de Vriesland. Óleo/lienzo adherido a cartón. Madrid. Museo del Prado.

AEA, LXXVIII, 2005, 311, pp. 297 a 331 
obra del pintor (siguiendo las directrices dadas por Aureliano de Beruete ${ }^{9}$ ), lo hizo, básicamente, en función de la evolución del colorido y la factura a lo largo de su producción, pero aquí, por razones evidentes, no podemos aplicar esos criterios. Aunque alguno de ellos, como veremos, nos puede valer. Lo que sí podemos aventurar, por lo pronto, a la vista del paisaje representado, tan llano, lacustre, cargado de vegetación y de clima lluvioso, que pudiera tratarse de algún paisaje de Bélgica, Holanda o del norte de Francia, captado por el pintor en alguna de las excursiones artísticas que a estos países hizo a lo largo de su vida. Si a ello unimos uno de los criterios que han servido para periodizar su pintura, como antes dijimos, veremos que podemos acercarnos, con cierta precisión, tanto al país de que se trata como más concretamente al lugar del mismo representado en el dibujo, así como también a los años por los que debió pintarse.

En efecto, en el verano de 1877, Haes emprende un viaje a Holanda, en compañía de su discípulo Jaime Morera, haciendo posiblemente un alto en las costas de Normandía ${ }^{10}$. Es este un momento clave en la vida privada y artística del pintor, ya que, a fines de 1876, sin haberse llegado a cumplir un año de su matrimonio, fallecieron su esposa y su hija de sobreparto. Esta desgracia, de la que nunca se recuperaría, determina la tercera y última etapa en la pintura de Haes, según la división que de su producción hizo Cid Priego ${ }^{11}$, como dijimos, y que se caracteriza por la utilización de una paleta más apagada, empleando tonalidades grisáceas, y que comienza a manifestarse en los paisajes marinos y lacustres de su brumosa tierra que pinta durante este viaje. Aquí, Haes se recrea "plasmando atardeceres y efectos lumínicos efímeros sobre las sosegadas aguas de las lagunas y tierras pantanosas del norte de Holanda (...). A este momento pertenecen los estudios pintados en las llanas y grisáceas tierras de Frisia, en los que la abundante vegetación, las lagunas y los canales ocupan una buena parte de su producción" 12 . El empleo de esta gama fría de colorido le sirve a Haes para la mejor expresión de la captación atmosférica, diluyendo así los fondos del cuadro y utilizando unos celajes «movidos y contrastados lumínicamente» ${ }^{13}$. Características todas estas que son perfectamente visibles en el dibujo que estudiamos. No tenemos más que sustituir la fría gama de colorido por el tono grisáceo obscuro del carboncillo, que vendría a ser su equivalente. Por lo demás, todas las características apuntadas para ese tipo de paisajes coinciden con las de este dibujo, como decimos: paisaje llano de lagunas con abundante vegetación, captación atmosférica, plasmación de efectos lumínicos efímeros y de atardecer sobre el paisaje y las aguas en particular, fondo de horizontes diluídos, así como cielos movidos y contrastados lumínicamente. Además, este gusto por la captación ambiental de los cambios atmosféricos fugaces, ese interés por representar la inestabilidad atmosférica, es característico de esta última etapa de la pintura de Haes, plasmándose de forma más concreta en las obras que realiza en Holanda y norte de Francia durante este viaje de 1877, climas ambos muy propicios a este tipo de fenómenos atmosféricos cambiantes. Todo ello, pues, nos lleva a pensar que, muy posiblemente, este dibujo fuese realizado por Haes durante su viaje a Holanda en 1877. Tomado, sin duda, del natural, posee esa frescura que da la inmediatez, aunque luego, probablemente, lo retocase en el taller, dado su perfecto acabado. Podemos, como consecuencia de lo expuesto, precisar que el asunto del dibujo es, con toda probabilidad, un paisaje tomado por Haes en tierras de Frisia, en el norte de Holanda, durante el viaje que allí realizó en 1877 , año que damos como su muy posible fecha de ejecución.

\footnotetext{
${ }^{9}$ Beruete, A. de. «Carlos de Haes», La Ilustración Española y Americana, año XLII, núm. XXIV, Madrid, 30 de junio de 1898, p. 275.

${ }^{10}$ Carlos de Haes en el Museo del Prado, Catálogo razonado a cargo de Ana Gutiérrez Márquez, Museo Nacional del Prado, Madrid, 2002, p. 40

${ }^{11}$ Cid Priego, C. Op. cit. nota 4, p. 22.

${ }^{12}$ Op. cit. nota 10, p. 41.

${ }^{13}$ Ibidem.
}

$A E A$, LXXVIII, 2005, 311, pp. 297 a 331 


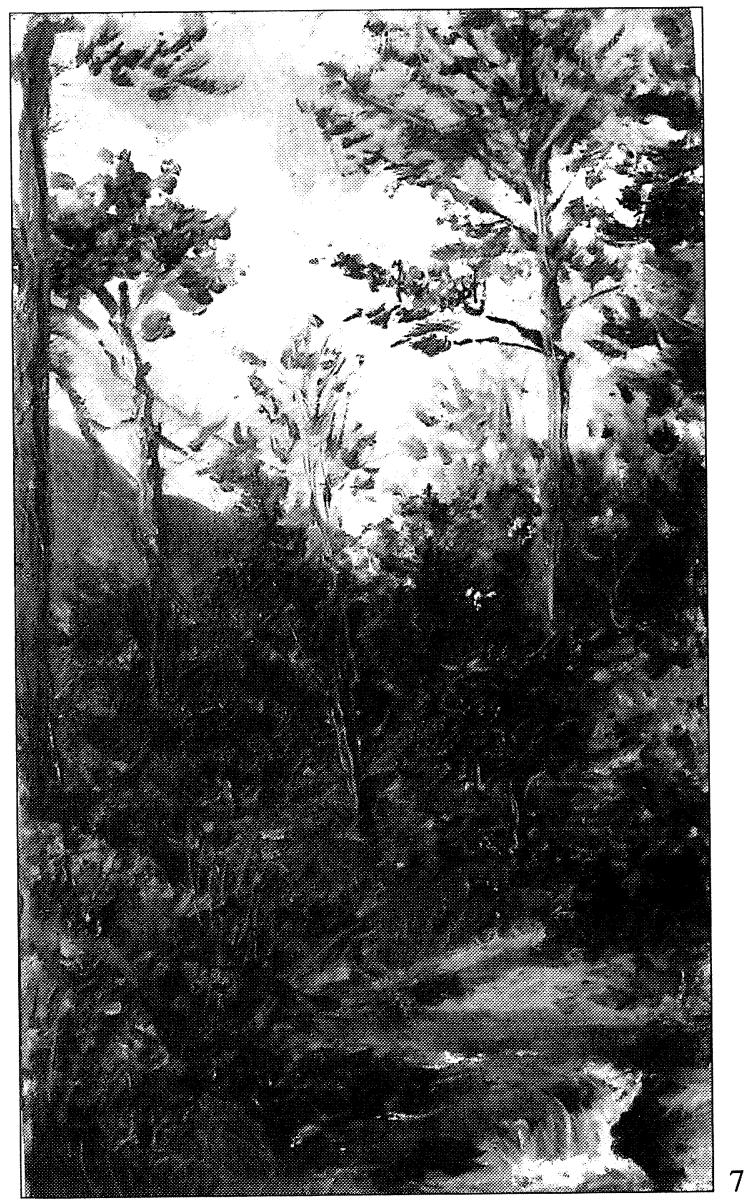

8

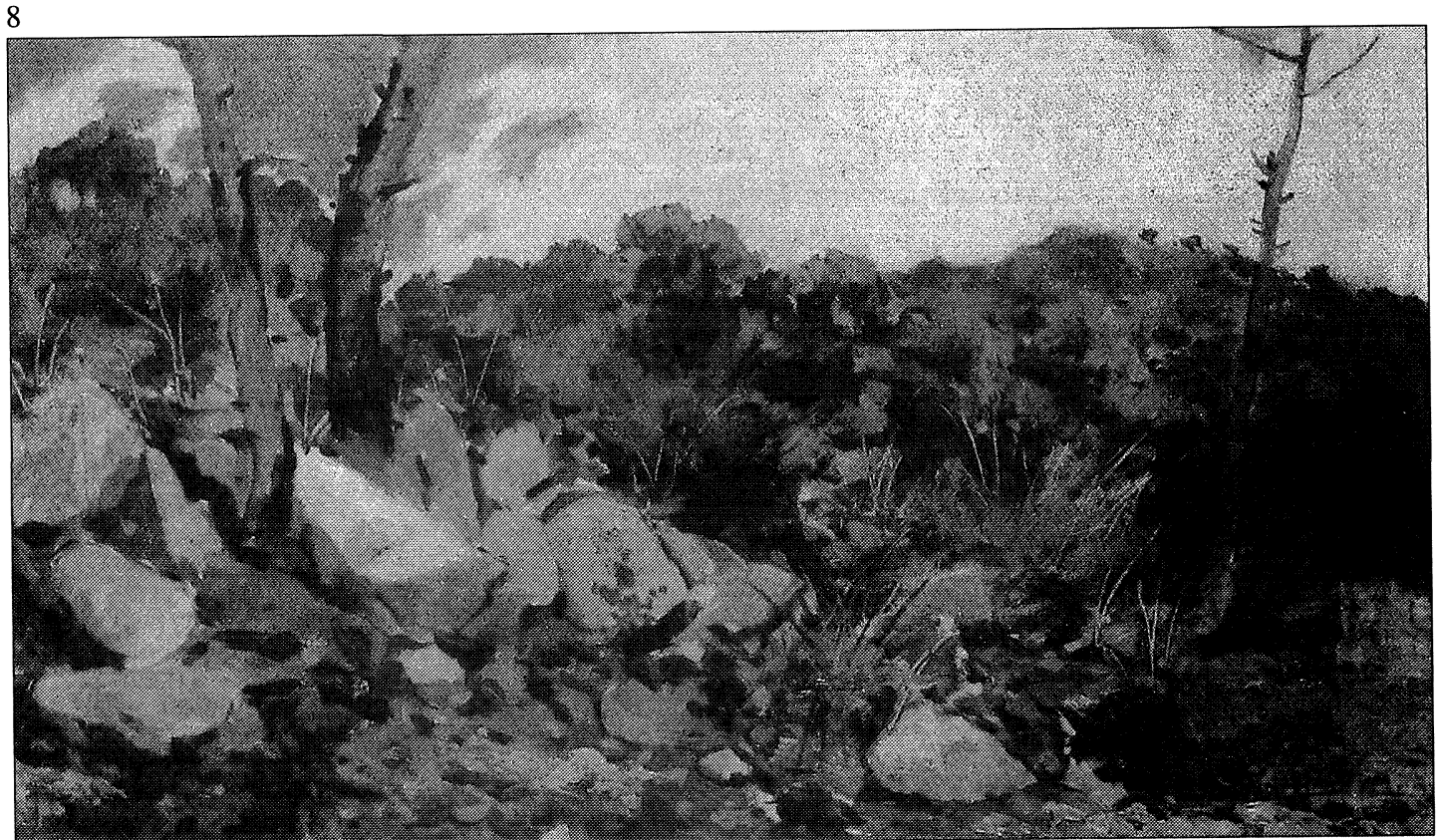

Fig. 7. Aureliano de Beruete: Paisaje de montaña. Óleo/tabla. Madrid. Colección particular.

Fig. 8. Aureliano de Beruete: Paisaje de la sierra madrileña. Óleo/tabla. Madrid. En el comercio.

AEA, LXXVIII, 2005, 311, pp. 297 a 331 
Pero, además de todo ello, podemos apoyar aún más nuestros argumentos comparando este dibujo con otras obras de Haes realizadas durante el citado viaje a Holanda en 1877, y que están perfectamente documentadas por pertenecer al Museo del Prado, casi todas procedentes de la generosa donación que al extinto Museo de Arte Moderno hicieron los discípulos de Haes en 1899. Así, por si nos quedara alguna duda, obras como Un canal de Vriesland ( ${ }^{\circ} 7056$ del Inventario General del Museo del Prado), datado hacia 1877 (Fig. 6); Laguna (Vriesland, Holanda) ( ${ }^{\circ} 5653$ del Inventario del Prado, depositado en el Museo de Arte Moderno de Barcelona), también datado hacia 1877; Lagunas de Abcoude (Holanda) (nº 7487 del Inventario del Prado, depositado en el Museo Jaime Morera de Lérida), igualmente datado hacia 1877; Cercanías de Abcoude (n 5777 del Inventario del Prado, depositado en el Museo de Cáceres), asimismo datado hacia 1877; o Un canal (Holanda) (registro del extinto Museo de Arte Moderno $86-\mathrm{H}$, actualmente en paradero desconocido), fechable igualmente hacia el mismo año ${ }^{14}$, muestran todas ellas tal similitud temática y estilística con el dibujo que estudiamos, que no sólo nos confirman la autenticidad de la obra, sino que, además, nos corroboran su temática como un paisaje de Frisia (Vriesland), e incluso su fecha de ejecución en 1877, durante el viaje que Haes realizó entonces a Holanda.

Respecto a los dos paisajes de Aureliano de Beruete que traemos, se trata de una tablita y de un lienzo de tamaño algo mayor que esa. Son obras que pertenecen a la primera etapa del pintor, cuando se hallaba aún bajo la influencia del arte de su maestro Carlos de Haes, antes, pues, de conocer a Martín Rico y a la escuela de Barbizón en París, influencias posteriores que, uniéndose a la de Haes, fueron las claves de gran parte de su producción, y a las que se sumarían más tarde los influjos del Impresionismo ${ }^{15}$.

Comenzaremos por la tablita, que es un hermoso óleo de $0,22 \times 0,13 \mathrm{~m}$. y que representa un Paisaje de montaña (Fig. 7), por el que discurre un riachuelo, cuyas aguas burbujean al primer término del cuadro, en su parte baja; una empinada ladera se ve al fondo izquierda de la composición, entre la vegetación y los árboles; las copas de éstos contrastan contra un celaje de intenso azul cargado de densas nubes blancas.

Aunque Beruete realizó numerosas excursiones artísticas, ya desde los inicios de su carrera pictórica, y muchas de ellas en compañía de Carlos de Haes, no sería de extrañar que este paisaje fuese de la sierra madrileña, ya que ésta y los alrededores de Madrid, en los que incidiría el pintor a partir de 1876 (dos años después de matricularse en la clase de Paisaje de la Escuela Especial de la Academia de San Fernando con Haes como maestro), fueron motivos preferentes de Beruete como producto de su relación con el círculo intelectual que se aglutinaba en torno al Colegio Internacional (Nicolás Salmerón y Francisco Giner de los Ríos), que comenzaban a reivindicar el paisaje madrileño y castellano con puntos referenciales en Velázquez y El Greco ${ }^{16}$.

Como decimos, es una bella tablita, cuya suelta y empastada factura, así como su riqueza de colorido, jugando fundamentalmente con una gama de verdes y pardos, sabiamente combinada, le proporcionan una sensación como de joyel. Por medio de la abundante y resaltada pasta el pintor juega con las luces y las formas, proporcionando volumen a los diferentes elementos vegetales, rayando en el empaste, de forma muy característica, para representar los alargados y finos troncos de los árboles. En la forma de dar la pasta, apreciamos claramente los pictogramas del artista, con su carácter espontáneo, de gran agilidad y, generalmente, de corto recorrido. La soltura de pincelada velazqueña parece querer estar aquí presente.

\footnotetext{
${ }^{14}$ Aunque, por razones de espacio, sólo reproducimos la primera de las obras citadas, a modo de ejemplo más representativo, todas las restantes se pueden ver reproducidas en Op. cit. nota 10, pp. 330, 334, 336, 338 y 346.

${ }^{15}$ Arias Anglés, E. Op. cit. nota 6, pp. 353 y 355.

${ }^{16}$ Ibidem, p. 353.
}

AEA, LXXVIII, 2005, 311, pp. 297 a 331 
La semejanza de esta tablita con otra de similares dimensiones $(0,24 \times 0,15 \mathrm{~m}$.), firmada y dedicada al maestro Mújica, que se subastó en junio de 2003 en la sala de subastas Durán, es bastante grande. Pero aún mayores pruebas de similitud, con esta que estudiamos, nos muestra otra tablilla con un Paisaje de la sierra madrileña (Fig. 8), que hace un tiempo se subastó en Ansorena (óleo/tabla, 0,13 x 0, 22 m., subasta $\mathrm{n}^{\circ} 239,2$ de octubre de 2001 , lote $n^{\circ} 34$ ), pues además de poseer las mismas dimensiones que ésta, aparecen también en ella representadas, de forma muy peculiar e inequívoca, unas hierbas de tallos largos y pelados, que en el cuadro que estudiamos se nos muestran a orillas del riachuelo a modo de juncos, y que se distribuyen entre las piedras, como si fueran espartos, en el cuadro de Ansorena. Estas finas varetas las ha realizado el pintor,

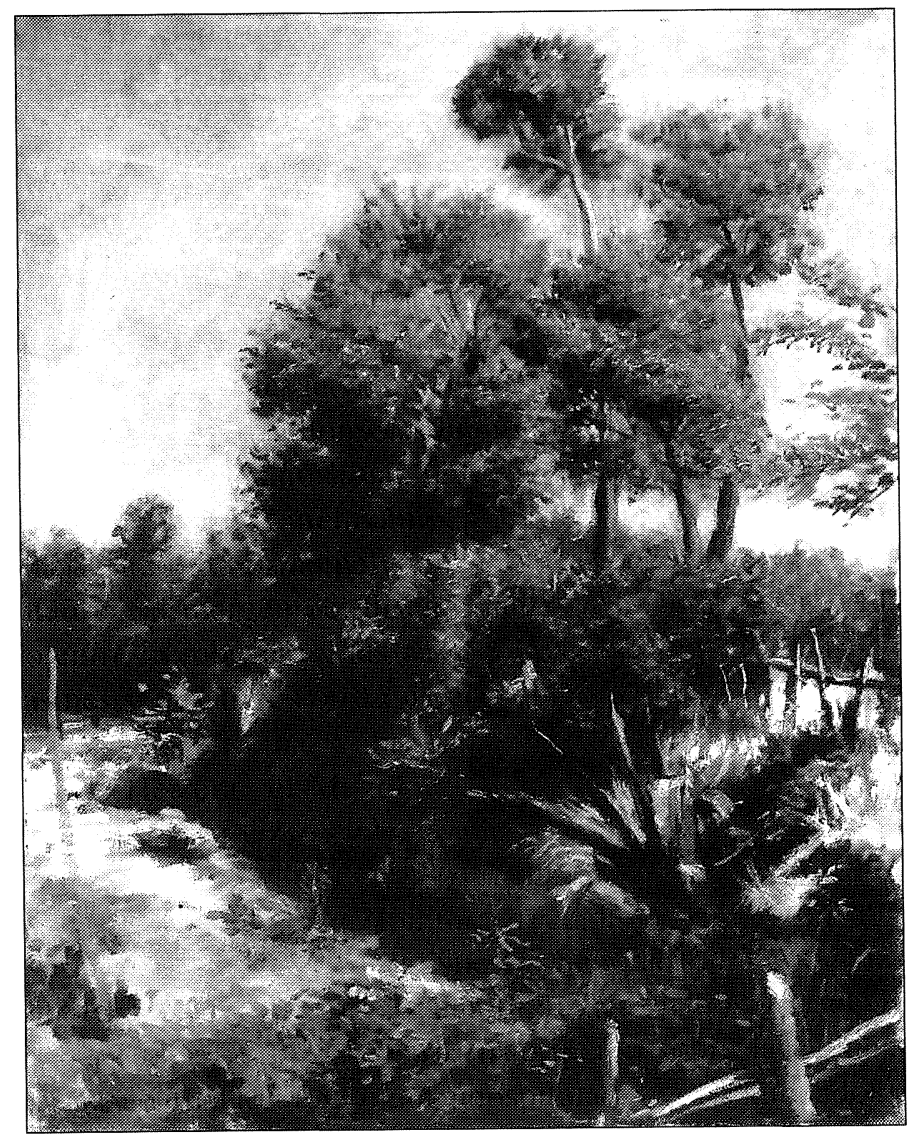

Fig. 9. Aureliano de Beruete: Paisaje campestre con pita. Óleo/lienzo. Madrid. Colección particular. en ambos cuadros, haciendo una suave incisión en la masa pictórica, a modo de ligero surco en la pasta, y vienen a ser como una especie de firma del artista puesta en los dos óleos, como una confirmación de la misma paternidad de ambos.

El otro cuadro de Beruete es un óleo sobre lienzo de $0,52 \times 0,41 \mathrm{~m}$., que representa un paisaje bastante característico del realismo, por lo que lo dataríamos también en la primera época del pintor, cuando aún se encontraba bajo la influencia de su maestro Carlos de Haes.

Lo titularemos Paisaje campestre con pita (Fig. 9), por ser este elemento vegetal el más destacado a primeros términos del cuadro y por ser, a su vez, el más destacable y conocido de las especies vegetales que pueblan y componen la obra. Es un bonito paisaje, aunque más elaborado y convencional frente a la gran espontaneidad y frescura que nos muestra el de la tablita que acabamos de analizar. Representa lo que parece ser la vista de un camino rural, que discurre desde los primeros términos, por el lateral izquierdo del cuadro, hacia el fondo de la composición; la parte central la ocupa un ligero terraplén cargado de diversa vegetación y árboles.

Pero a pesar de su acusada fisonomía realista, la factura es suelta, empastada, y de corto pictograma, a semejanza de como acabamos de ver en la tablita que antes hemos analizado. Igualmente, los efectos lumínicos, los juegos de luces y sombras, tamizados por la vegetación sobre el suelo, la forma de proyectar la luz, se corresponde con la misma manera de utilizarla en numerosas de las obras de Beruete de este período, antes de su etapa que lo acerca al Impresionismo. Igualmente la utilización de un cielo liso y límpido de fuerte azul, tiene su equi- 


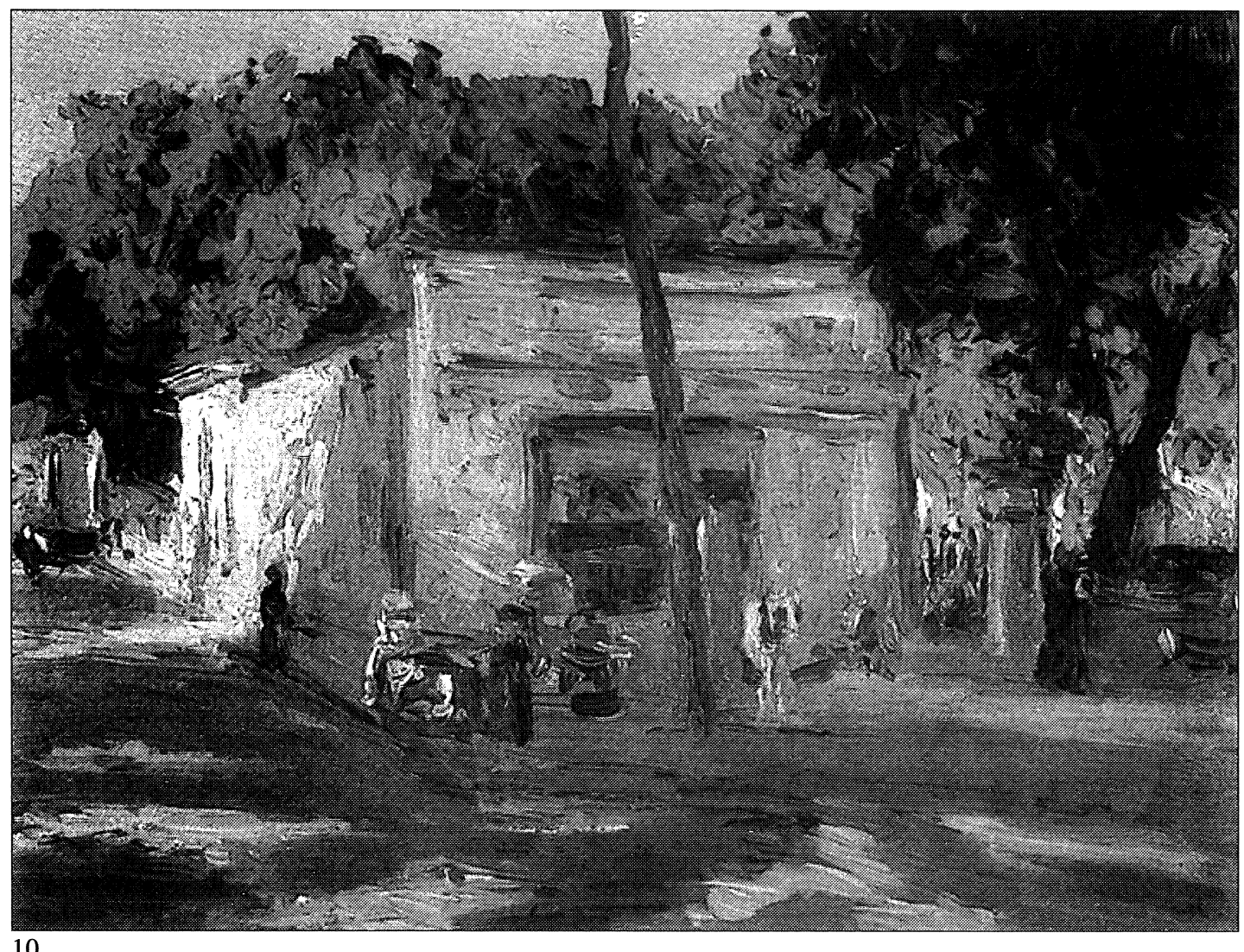

10

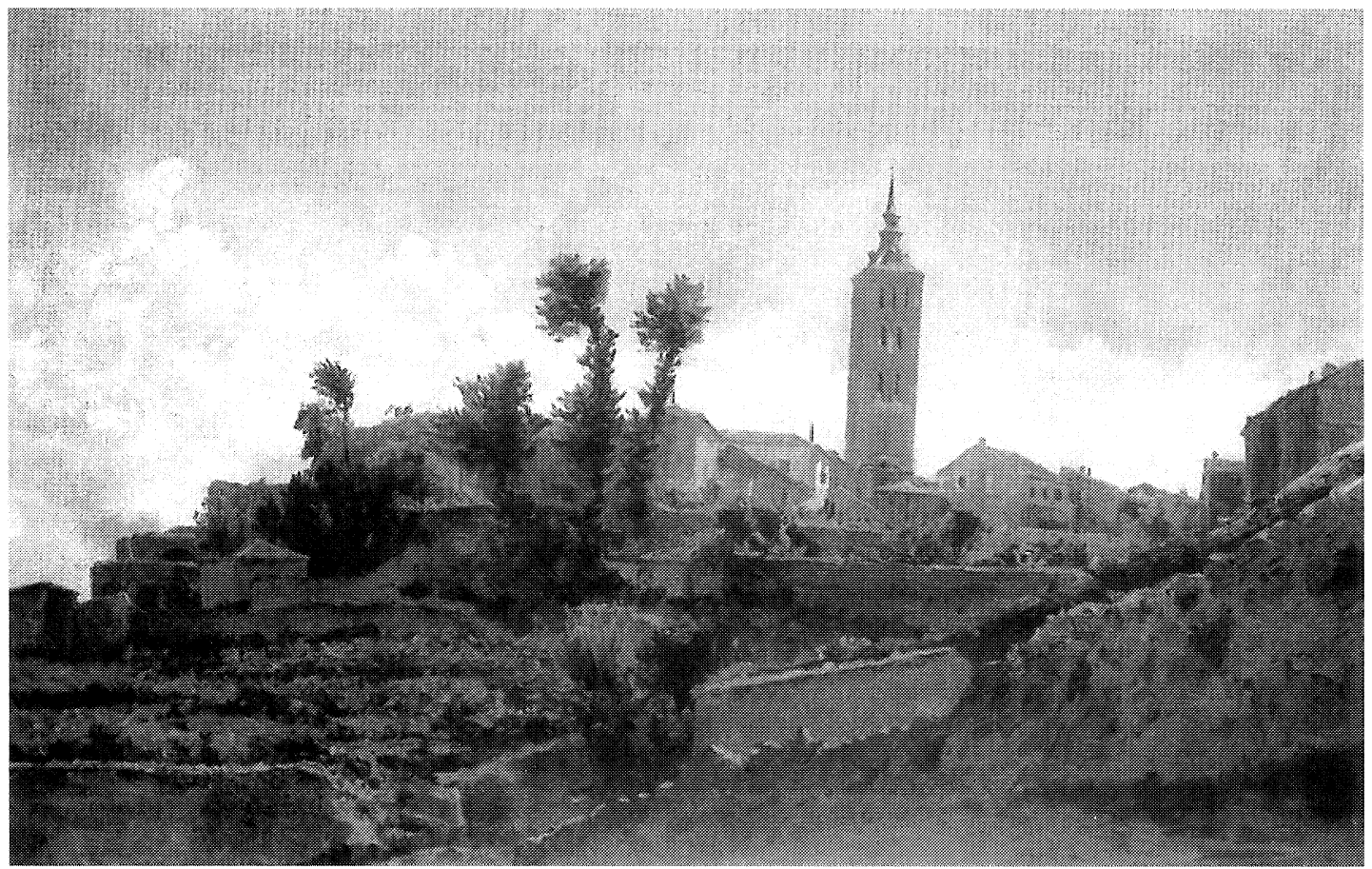

Fig. 10. Aureliano de Beruete: Vichy. Óleo/cartón. Madrid. Colección particular.

Fig. 11. Aureliano de Beruete: Paisaje de Segovia. Óleo/lienzo. Madrid: Colección de Arte de Caja Madrid.

$A E A$, LXXVIII, 2005, 311, pp. 297 a 331 
valente en otras obras del artista, como El asilo de San Bernardino de Madrid (Museo Municipal. Madrid) o la Vista de la Casa de Campo (óleo/lienzo de 0,50 × 0,69 m. subastado en Durán en febrero de 2002, lote 222), por citar algún ejemplo. Y lo mismo podemos decir de la factura de los árboles, con sus característicos y esbeltos troncos, realizados a base de largas rayas sobre el empaste, patente en cuadros como Vichy (Fig. 10) ${ }^{17}$ (óleo/cartón, 0,255 x 0,34 m., colección particular. Madrid), de gran semejanza con los alargados y rayados troncos del cuadro que estudiamos; pero no sólo son los troncos, sino también la factura de las copas de los árboles las que se convierten en otro referente de identidad si las comparamos, por ejemplo, con las representadas en cuadros como Vista de Toledo (Museo de Arte Moderno. Barcelona), de hojarasca de factura tan igual con la del cuadro que estudiamos; o si nos fijamos en la forma tan peculiar de terminar las copas de los árboles en cuadros como Vista del sur de Toledo desde los Cigarrales (Museo de Arte Contemporáneo. Toledo) o la Vista de la Vega Baja desde El Cambrón: el río y sus riberas con la fábrica de armas al fondo, del mismo Museo, o Paisaje de Segovia (Fig. 11) (óleo/lienzo, 0,40 × 0,60 m., Colección de Arte de Caja Madrid), tan semejantes a la manera como se rematan las copas de los árboles en este cuadro que presentamos. Todo ello, como podemos ver, son elementos comunes de identidad, presentes tanto en este cuadro como en esas y otras obras de Aureliano de Beruete.

ENRIQUe ARIAS ANGLÉS I. Historia (CSIC)

\section{EL RETRATO DE LA ÚLTIMA HIJA DE JUAN BAUTISTA MARTÍNEZ DEL MAZO, BOCETO PARA EL RETRATO DE LA FAMILIA DEL PINTOR DEL KUNSTHISTORISCHES MUSEUM DE VIENA}

Por adquisición en el comercio de antigüedades francés se conserva en una colección particular de Bilbao el Retrato de una niña (fig. 2), pintura de carácter velazqueño, que puede ser clasificada perfectamente como obra indiscutible del pintor Juan Bautista Martínez del Mazo (¿Beteta?, Cuenca, 1611-Madrid, 1667), tanto por la característica soltura técnica, como por tratarse de uno de los modelos infantiles que aparecen en el Retrato de la familia del pintor del Kunsthistorisches Museum de Viena (fig. 3), en relación con la cual se conoce otro Retrato de Luis Martínez del Mazo conservado en la Dulwich Picture Gallery de Londres (fig. 1) ${ }^{1}$. La importancia de la obra es notable no sólo porque incrementa el catálogo de Martínez del Mazo con una obra indudable, sino también por contribuir a precisar la cronología de la pintura conservada en Viena ${ }^{2}$.

\footnotetext{
${ }^{17}$ Ibidem, pp. 422-423.

${ }^{1}$ Esta pintura ya aparece recogida en la segunda edición de 1903 de la obra Carl Justi, Velázquez y su siglo. Madrid, 1953, p. 774. Se reproduce en el Velázquez de los Klassiker der Kunst, Stuttgard y Berlín, 1913, p. 229. Juan Antonio Gaya Nuño. La pintura española fuera de España. Historia y catálogo. Madrid, 1958, p. 230, $\mathrm{n}^{\circ} 1727$. Su reproducción puede verse en el libro de José López-Rey Velázquez, Painter of painters. Colonia, Taschen - Wildenstein Institute, 1996, vol. I, p. 190. El niño retratado en el lienzo de Londres puede ser identificado con don Luis del Mazo y de la Vega, segundo hijo del matrimonio del pintor con Francisca de la Vega (Vid. infra). Así se recoge en la obra de Richard Beresford, Dulwich Picture Gallery. Completed Illustrated Catalogue. Londres, 1998, p. 157.

${ }^{2}$ Bibliografía básica sobre el pintor que se citará en las notas es la siguiente: $\mathrm{M}^{\mathrm{a}}$ Luisa Caturla, «Sobre un viaje inédito de Mazo a Italia, hasta ahora ignorado», en Archivo Español de Arte, 1955, pp- 7-75. José López Navío. «El matrimonio de Juan Bautista Martínez del Mazo con la hija de Velázquez», en Archivo Español de Arte, xxxiI, 1960, pp. 389-419. Varia velazqueña. Homenaje a Velázquez en el III Centenario de su muerte. 1660-1960. Volumen II. Elogios poéticos. Textos y comentarios críticos. Documentos. Cronología. Láminas e índices. Madrid, 1960. Desde el documento 209 en adelante. Juan Antonio Gaya Nuño, «Juan Bautista del Mazo el gran discípulo de Velázquez», en Varia Velazqueña. Home-
} 